\title{
Modelos de gestión de conflictos en serie de ficción televisiva
}

\section{Conflict management models in television fiction series}

\author{
Yolanda Navarro-Abal ${ }^{1}$, José Antonio Climent-Rodríguez ${ }^{1}$ \\ y Julio Fernández- Garrido ${ }^{2}$ \\ ${ }^{1}$ Departamento de Psicología Clínica, Experimental y Social, Universidad de Huelva, España \\ ${ }^{2}$ Departamento de Personalidad, Evaluación y Tratamiento Psicológico. Universidad Complutense de Madrid, España
}

Disponible online 31 de diciembre de 2012

\begin{abstract}
Las series de ficción televisivas generan, en ocasiones, una visión irreal de la vida, especialmente en los jóvenes, convirtiéndose en un espejo en el que se sienten reflejados. Se convierten en modelos de valores, actitudes, habilidades y comportamientos que tienden a ser imitados por ciertos espectadores. El objetivo del presente estudio ha sido analizar los estilos de gestión conductual de los conflictos escenificados por los protagonistas principales de las series de televisión de ficción. Para ello, se valoró la relación existente entre dichos estilos y la edad y el sexo de los protagonistas principales, así como la nacionalidad y el género de las series de ficción. Se han evaluado 16 series de ficción, seleccionando en cada una dos protagonistas (de ambos sexos). Para la observación y el registro se realizó una adaptación del Rahim Organizational Conflict Inventory-II. Los resultados reflejan que no existe una relación de los estilos de gestión conductual de los conflictos escenificados en las series de ficción y el sexo de los protagonistas. Se hallan relaciones entre dichos estilos y la edad de los protagonistas y el género de las series de ficción.
\end{abstract}

Palabras clave: Estilos de Gestión de Conflictos; Series de Ficción; Influencia; Modelado; Socialización.

Television fiction series sometimes generate an unreal vision of life, especially among young people, becoming a mirror in which they can see themselves reflected. The series become models of values, attitudes, skills and behaviours that tend to be imitated by some viewers. The aim of this study was to analyze the conflict management behavioural styles presented by the main characters of television fiction series. Thus, we evaluated the association between these styles and the age and sex of the main characters, as well as the nationality and genre of the fiction series. 16 fiction series were assessed by selecting two characters of both sexes from each series. We adapted the Rahim Organizational Conflict Inventory-II for observing and recording the data. The results show that there is no direct association between the conflict management behavioural styles presented in the drama series and the sex of the main characters. However, associations were found between these styles and the age of the characters and the genre of the fiction series.

Keywords: Conflict management styles; television fiction series; influence; modelling; socialization.

Correspondencia: Yolanda Navarro Abal. Departamento de Psicología Clínica, Experimental y Social. Universidad de Huelva. Tf.: 959219544/9099. E-mail: yolanda.navarro@dpsi.uhu.es. E-mail de los otros autores: José A. Climent Rodríguez: jose.climent@dpsi.uhu.es. Julio Fernández Garrido: juliofg@psi.ucm.es 
Muchos han sido los estudios que, desde el nacimiento del medio televisivo, han intentado comprender la influencia de esta poderosa herramienta de trasmisión y recepción de comunicación en el proceso de socialización de la persona, llegándose a consensos en cuanto a que sus efectos no dependen en exclusiva de las características de los contenidos sino también de las características de la audiencia (Sahuquillo, 2007). La influencia del medio televisivo en la socialización de los niños y niñas del último medio siglo resulta evidente, aportando elementos de aculturación y trascendiendo a barreras históricas, así como proporcionando elementos novedosos de comparación de contextos y realidades diversas, e introduciendo nuevas formas de interrelación y de interacción con el entorno (Lucas, García y Ruiz, 1999; Thompson, 1998).

El modo y la forma en la que se produce la interacción entre el medio televisivo y la persona ha tratado de explicarse a través de las teorías del aprendizaje social, así como desde otros modelos aportados por la psicología social. De esta forma, y tal como señala Huertas (2002), la teoría del Aprendizaje Vicario de Bandura (1977) surgió para estudiar el efecto de la violencia en televisión sobre la conducta. Diversos autores (Ball-Rokeach y DeFleur, 1976; Del Río, Alvarez y Del Río, 2004; Lucas, García y Ruiz, 1999; Jeffres, 1986; Sauquillo, 2007; Wimmer y Dominick, 1996) han corroborado esta teoría, incidiendo en diferentes aspectos en cuanto a las causas que provocan el modelado simbólico de los medios televisivos.

Las series de ficción son un género televisivo destinado al entretenimiento de las audiencias mediante relatos inventados, cuya distribución enlatada posibilita su programación en diferentes franjas horarias (Carrasco, 2010).

Existe una gran controversia en cuanto a la clasificación de los diferentes géneros televisivos, encontrándose una gran variedad de clasificaciones, y a su vez, una gran variedad de subgéneros en las propias series de ficción (Sánchez-Noriega, 2010). Así por ejemplo, se pueden distinguir categorizaciones en relación a los contenidos, tales como policiacas, drama o comedia, o bien algunas clasificaciones llevadas a cabo en función del receptor al que va dirigido, como es el caso de las teen series, que tal como indican García- Muñoz y Fedele (2011), están dirigidas al target juvenil y muestran una determinada realidad juvenil en contextos o escenarios específicos a la espera de captar la atención del público joven.

Constituyen un fenómeno social de gran influencia, sobre todo en jóvenes (Pasquier, 1996), y que han tenido, y siguen teniendo, una indudable repercusión en la construcción de la identidad en la infancia y en la adolescencia. Potter (1990) encontró que cuanto más series de ficción veían los estudiantes de EE UU, más valores convencionales de la clase media norteamericana poseían. Durkin (1995), França (2000), y Huston-Stein y Frederick (1972) señalan que los personajes de las series de ficción son modelos para adolescentes e influyen en su comportamiento, buscando nuevos modelos alejados del entorno familiar, siendo un referente mayor para ellos que la vida real (Feilitzen y Linne, 1975; Joy, Kimball y Zabrack, 1986), sobre todo cuando hay carencia o ausencia de figuras familiares de referencia (Nimmo, 1978).

Los roles y estereotipos de los personajes, de las series de ficción, son un prolífico objeto de estudio debido, sobre todo, a la preocupación en situar el foco de antecedente o consecuente en los mass-media, o en la propia sociedad, en relación a esas conductas estereotipadas y reflejadas en la televisión (Gunter, 1986; Heintz-Knowles, 2000; Lauzen, Dozier y Horan, 2008). Martínez y Surinvac (1998) argumentan que el uso frecuente de los estereotipos se debe a su funcionalidad, actuando como un simplificador de los atributos psicológicos de los personajes, que permiten la rápida y fácil comprensión por el espectador en el limitado "tempo" televisivo. De otra parte, Loscertales y Nuñez (2009) señalan que los estereotipos mostrados en los medios audiovisuales pueden ser de rol, esto es, lo prohibido y lo permitido para cada grupo, o de rasgo, más consistentes y elementales, que asignan rasgos de personalidad diferenciados a mujeres y hombres, jóvenes y adultos, personas de diferentes culturas, etc, y que pueden ser identificados por los adjetivos usados en sus descripciones o interacciones.

En cuanto a diferencias de género y su representación en ficción seriada, cabe destacar el estudio sobre series españolas realizado por Galán (2007), en el que destaca que las mujeres y hombres, tienen un mayor atractivo físico cuanto mayor sea el rango profesional que ocupan como personaje en la serie. Así, aunque se evidencia una relación de igualdad en el contexto laboral donde se desarrolla la trama, se aprecia que ellas tienen mayor preocupación por asuntos familiares y personales que sus compañeros masculinos. También, se representa al personaje femenino con conductas más inseguras frente a los personajes masculinos, que se muestran con comportamientos más amenazantes, agresivos, violentos e irónicos, con gran eficacia profesional y más resolutivos en la gestión de los problemas.

Otros autores han establecido clasificaciones más o menos detalladas sobre estereotipos de mujeres y hombres en las "sitcom" (Bernárdez, García y González, 2008; Guarinos 2009; Toledano y Verde, 2007). Los personajes femeninos a menudo están menos representados, o representados en número inferior, respecto a los personajes masculinos. Además, las mujeres representadas tienden a ser más jóvenes que los hombres (Signorielli y Bacue 1999), y en posiciones o actitudes más débiles o sumisas, mientras que los hombres tienden a ser más fuertes y poderosos (Mastro y Ortiz, 2008).

En relación al sexo y teniendo en cuenta el cada vez mayor auge de las series de "género" (entendidas como las orientadas a un público masculino o femenino), Simelio (2010) apunta que las tramas de ficción televisiva sobre mujeres son mayoritariamente referidas a relaciones personales y sentimentales, mientras que las tramas laborales son protagonizadas por los varones.

Con respecto a estereotipos relacionados con la edad, estudios como el de López y Cuenca (2005) han determinado 
que las series de ficción de producción nacional proyectan una imagen estereotipada de tercera edad a través de las personas mayores que aparecen en ellas, centrada en las ideas de consejero experto y protector de la familia. Por su parte, el estudio de Guarinos (2009) realiza una comparación de estereotipos de adolescencia en series españolas y americanas, concluyendo que las series nacionales construyen un perfil de adolescente más duro, y más cercano a la realidad, mientras que el modelo americano se centra en construir un universo simbólico e irreal e imposible, que no es más que la representación de los valores morales de esa cultura (Digón, 2006).

En cuanto a la representación de los conflictos, simbolizados habitualmente como actos violentos, y su influencia en la conducta, numerosos estudios (Bryant y Zillmann, 2002; Cheung y Chan, 1996; Ward, 2003) destacan el aumento de estas conductas antisociales en personas que ven habitualmente escenas de violencia en televisión. También, se ha señalado que cuando la violencia es injustificada decrecen las actitudes agresivas de los sujetos estudiados (Berkowitz y Powers, 1979; Hoyt, 1970; Krcmar y Cooke, 2001; Palmer y Dorr, 1980). Por su parte, investigaciones ya clásicas referidas al discurso narrativo de las series de televisión (Raffa, 1983) y su influencia en el desarrollo moral, concluyen que los valores antisociales se presentan con más intensidad que los positivos, sin embargo, también expone que en los contenidos televisivos están presentes las conductas prosociales, como por ejemplo, la resolución de conflictos por medios no violentos. En este sentido, Gale (2001) analiza los valores de series norteamericanas de gran audiencia y su influencia en jóvenes, destacando la transmisión de valores positivos relacionados con la cooperación, la no acomodación ante los problemas o la responsabilidad personal en relación a las estrategias de gestión de conflictos más frecuentemente utilizadas por parte de los personajes protagonistas.

Los estilos conductuales para gestionar conflictos no son más que el resultado de la interacción entre nuestras características de personalidad individuales y aquellas habilidades que hayamos adquirido a lo largo de nuestro proceso de socialización. Para su distinción, Rahim y Bonoma (1979) realizaron una clasificación basada en dos dimensiones fundamentales (interés por sí mismo e interés por los demás). De la interacción de estas dimensiones plantean una clasificación en donde diferencian cinco categorías que son: compromiso, integración, evitación, servilismo y dominación. Cada uno de ellos será usado en mayor medida en función de muchas variables, entre ellas, la situación que la persona afronte, o bien, el estilo que haya adquirido a lo largo de su proceso educacional y de socialización. Cabe destacar la importancia que cobran mecanismos de modelado y aprendizaje vicario para el aprendizaje e integración de unos u otros modelos de gestión conductual del conflicto, propios de cada persona. Los estilos familiares, los modelos educativos, la influencia de iguales, pero también la exposición prolongada a modelos de ficción, y entre estos últimos, los más significativos son los aportados por el medio televisivo, tienen relación con el estilo o estilos de gestión de conflictos predominante, sobre todo, en el niño y en el joven.

El objetivo general de este trabajo es analizar los modelos preferentes de estilos de gestión de los conflictos manifestados por los protagonistas principales de las series de ficción de las cadenas nacionales generalistas de España. En relación a este objetivo general, se plantearon cuatro objetivos específicos que son:

a) Analizar si existe relación entre los diferentes estilos de gestión de conflictos escenificados por los protagonistas principales y el sexo de los mismos.

b) Valorar si existe relación entre los diferentes estilos de gestión de conflictos escenificados por los protagonistas principales y la edad de los mismos.

c) Distinguir si existe relación entre los diferentes estilos de gestión de conflictos escenificados por los protagonistas principales y la nacionalidad de la serie de ficción. Para la realización de este estudio se han reducido en dos categorías básicas: series de nacionalidad española, producidas por una productora española o en formato de coproducción pero con una mayoría (más del 75\%) de producción española y, series de nacionalidad extranjera, cuando no se cumpla el requisito anteriormente expuesto.

d) Observar si existe relación entre los diferentes estilos de gestión de conflictos escenificados por los protagonistas principales y el género de la serie de ficción.

\section{Método}

\section{Muestra}

Para la realización de este trabajo se seleccionaron 32 personajes de ambos sexos (16 hombres y 16 mujeres), que eran los protagonistas principales de las series de ficción visionadas. Para ello se seleccionaron 16 series de ficción, correspondiente a series que se retransmitían en las cadenas de televisión de carácter generalista y de ámbito nacional. Las cadenas visionadas fueron: TVE1, TVE2, Antena3, Telecinco, la Sexta y Cuatro.

\section{Instrumentos de evaluación}

Para la observación y registro se realizó una adaptación del Inventario Rahim Organizational Conflict Inventory-II (ROCIII) (Rahim, 1983, validación española Munduate, Ganaza y Alcaide, 1993). El instrumento consta de 28 ítems, distribuidos en cinco escalas de estilos de gestión conductual de conflictos que son integración, compromiso, dominación, evitación y servilismo, que se responden según una escala tipo Likert de 5 puntos. Presenta un coeficiente total de Cronbach para la escala total de .78: integración (.77); compromiso (.62); dominación (.75); evitación (.70); servilismo (.76).

\section{Procedimiento}

Para la adecuada realización de un análisis de contenido con carácter científico es necesario llevar a cabo una rigurosa recogida de información que sea sistemática, objetiva, replicable y 
válida. Para cumplir estos objetivos, los investigadores de este estudio realizaron los registros mediante el ROCI-II (adaptado para este trabajo). Dicho instrumento ha mostrado indicadores de fiabilidad y confianza. Se visionaron, a lo largo de tres meses (de enero a marzo de 2012), un total de 16 series de ficción de los canales de televisión con carácter generalista, es decir, TVE 1, TVE 2, Antena3, Telecinco, la Cuatro y la Sexta. Para su visionado, se grabaron durante los meses de octubre, noviembre y diciembre de 2011, un capítulo cada mes. Este procedimiento evitaba posibles sesgos del observador cuando realizaba el análisis de los conflictos, derivados de la menor objetividad, bien por simpatía o por rechazo, de los personajes cuando se conocía la trama de la serie de una forma continua (capítulo a capítulo). La selección de la series, y de sus protagonistas, se llevaron a cabo en función a una encuesta realizada por los autores sobre series más visionadas por edades. Ésta formaba parte de una batería de preguntas correspondiente a un proyecto de investigación que se encuentra en desarrollo. La recogida de información se realizó en una muestra conformada por un total de 170 jóvenes, 123 (alumnado de la Universidad de Huelva) y, 47 (alumnado de un Instituto de Secundaria de la misma ciudad). La ratio de edad estuvo conformada entre los 17 y 22 años. Una vez registradas las series que eran de interés para el estudio, se clasificaron en función a cuatro géneros, que fueron, los dos tradicionales, drama y comedia, y policíacas y teen age.

Las series seleccionadas y sus protagonistas fueron: La fuga (Anna y Daniel); Toledo (Paula y Martín); El Barco (Ainhoa y Ulises); Bones (Temperance Brennan y Seeley Booth); Mentalista (Teresa Lisbon y Patrick Jane); Bandolera (Sara Reeves y Héctor Ibáñez); Tierra de lobos (Almudena y César); Cuéntame como pasó (Inés y Toni); Con el culo al aire (Sandra y Tino); Navy (Ziva David y Tony Dinozzo); Los protegidos (Sandra y Culebra); Puente Viejo (Pepa y Tristán); Revenge (Emily Thorne y Daniel Grayson); Aída (Soraya y Luisma); Mentes Criminales (Derek Morgan y Jennifer 'JJ'); CSI Las Vegas (Gil Grissom y Catherine Willows).

\section{Análisis de datos}

Se procedió al análisis de los resultados mediante el paquete estadístico SPSS (versión 19.0). El análisis estadístico se realizó en función de los objetivos planteados así como a las características de las variables objetos de estudio, que fueron: por un lado, se realizó un análisis descriptivo (media y desviación típica) de las variables de estudio, por otro lado, se llevó a cabo un análisis de varianza (ANOVA) de un factor para comparar los diferentes estilos de gestión conductual de los conflictos en función a las variable edad y sexo de los protagonistas principales, nacionalidad y género de la series de ficción (con análisis post hoc mediante el método Sheffé).

Los estilos de gestión de los conflictos estudiados se encuentran determinados por las escalas que componen el propio instrumento de evaluación, es decir, integración, compromiso dominación, evitación y servilismo.

\section{Resultados}

En primer lugar, se analizan los Estilos de Gestión Conductual de los Conflictos en relación al sexo de los protagonistas (tabla 1). Como se puede observar, la distribución $F$ de FisherSnedecor no muestra diferencias significativas en las diferentes escalas y el sexo de los protagonistas.

En la tabla 2 se detallan los resultados del ANOVA obtenidos en relación a la edad de los protagonistas de la serie. Como se observa, existen diferencias estadísticamente significativas para cada una de las dimensiones de los estilos.

Una vez aplicada la prueba Sheffé (tabla 3 ) se observa que los grupos muestrales que presentan diferencias significativas son: en relación al estilo de gestión conductual de integración, entre los personajes adolescentes y las personas mayores, observando que son las personas mayores $(M=26.71)$ las que obtienen los resultados más elevados frente a los adolescentes $(M=16.22)$, que obtienen el menor valor; en cuanto al estilo de evitación, los resultados indican diferencias estadísticamente significativas entre los adolescentes con adultos y

Tabla 1

Estilos de Gestión del Conflicto en función del Sexo de los protagonistas.

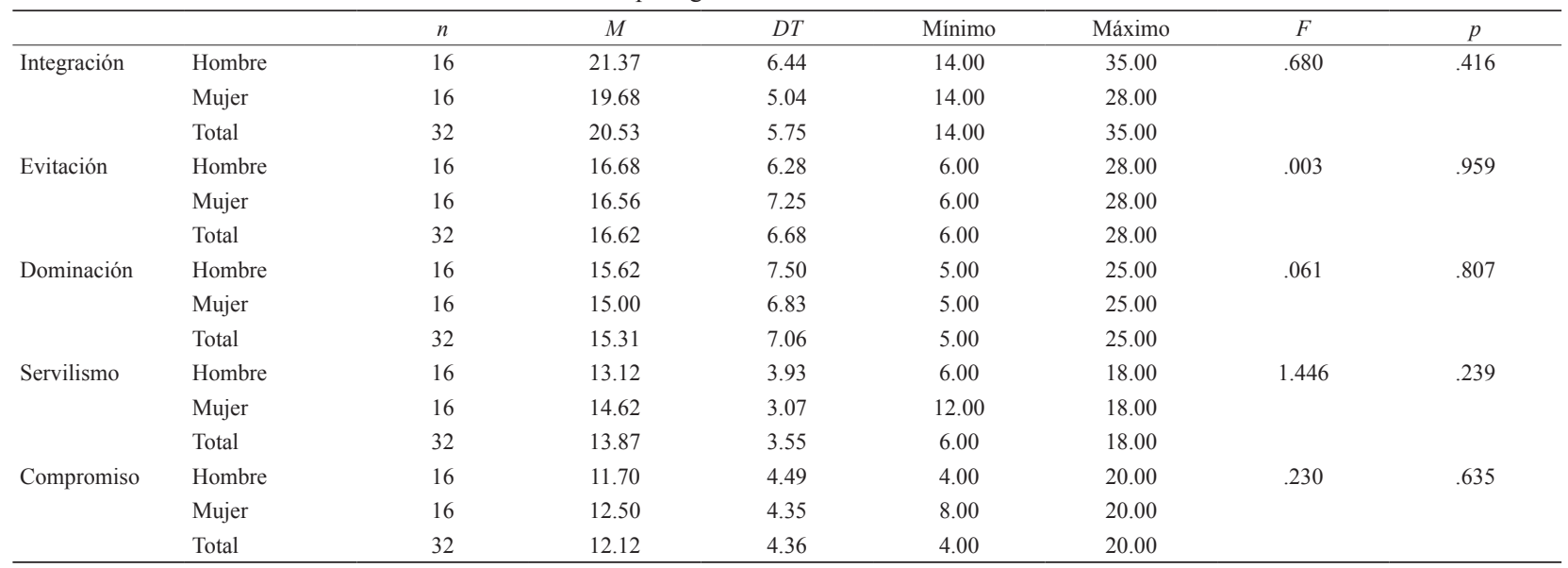


MODELOS DE GESTIÓN DE CONFLICTOS

Tabla 2

Estilos de Gestión del Conflicto en función de la Edad de los protagonistas.

\begin{tabular}{|c|c|c|c|c|c|c|c|c|}
\hline & & $n$ & $M$ & $D T$ & Mínimo & Máximo & $F$ & $p$ \\
\hline \multirow{5}{*}{ Integración } & Adolescencia & 9 & 16.22 & 2.58 & 14.00 & 21.00 & 8.96 & .000 \\
\hline & Joven & 5 & 17.20 & 3.96 & 14.00 & 22.00 & & \\
\hline & Adultez & 11 & 21.63 & 4.90 & 14.00 & 28.00 & & \\
\hline & Vejez & 7 & 26.71 & 5.28 & 19.00 & 35.00 & & \\
\hline & Total & 32 & 20.53 & 5.75 & 14.00 & 35.00 & & \\
\hline \multirow[t]{5}{*}{ Evitación } & Adolescencia & 9 & 10.11 & 4.40 & 6.00 & 17.00 & 22.38 & .000 \\
\hline & Joven & 5 & 11.80 & 3.56 & 7.00 & 17.00 & & \\
\hline & Adultez & 11 & 19.36 & 3.55 & 13.00 & 23.00 & & \\
\hline & Vejez & 7 & 24.14 & 3.53 & 18.00 & 28.00 & & \\
\hline & Total & 32 & 16.62 & 6.68 & 6.00 & 28.00 & & \\
\hline \multirow[t]{5}{*}{ Dominación } & Adolescencia & 9 & 22.77 & 2.63 & 20.00 & 25.00 & 41.85 & .000 \\
\hline & Joven & 5 & 21.00 & 2.23 & 20.00 & 25.00 & & \\
\hline & Adultez & 11 & 11.81 & 4.04 & 5.00 & 20.00 & & \\
\hline & Vejez & 7 & 7.14 & 2.67 & 5.00 & 10.00 & & \\
\hline & Total & 32 & 15.31 & 7.06 & 5.00 & 25.00 & & \\
\hline \multirow[t]{5}{*}{ Servilismo } & Adolescencia & 9 & 12.00 & .00 & 12.00 & 12.00 & 5.25 & .005 \\
\hline & Joven & 5 & 10.80 & 2.68 & 6.00 & 12.00 & & \\
\hline & Adultez & 11 & 15.27 & 3.13 & 12.00 & 18.00 & & \\
\hline & Vejez & 7 & 16.28 & 4.53 & 6.00 & 18.00 & & \\
\hline & Total & 32 & 13.87 & 3.55 & 6.00 & 18.00 & & \\
\hline \multirow[t]{5}{*}{ Compromiso } & Adolescencia & 9 & 7.55 & 2.40 & 4.00 & 12.00 & 13.53 & .000 \\
\hline & Joven & 5 & 11.20 & 1.78 & 8.00 & 12.00 & & \\
\hline & Adultez & 11 & 13.45 & 3.69 & 8.00 & 20.00 & & \\
\hline & Vejez & 7 & 16.57 & 2.76 & 12.00 & 20.00 & & \\
\hline & Total & 32 & 12.12 & 4.36 & 4.00 & 20.00 & & \\
\hline
\end{tabular}

con personas mayores, y este resultado también aparece en los jóvenes. El grupo que obtiene una mayor puntuación en este estilo es el de las personas mayores $(M=24.14)$, en oposición al de menor valor que es el de adolescentes $(M=10.11)$; en relación al estilo de gestión conductual de dominación, las diferencias halladas se encuentran entre el grupo de adolescentes con adultos y personas mayores, jóvenes con adultos y personas mayores y, a su vez, adultos con personas mayores, siendo en esta ocasión obtenida la puntuación más elevada en el grupo de adolescentes $(M=22.77)$, frente a la de menor puntuación conseguida por las personas mayores $(M=7.14)$; en relación al estilo de gestión conductual de servilismo, aparecen diferencias significativas entre el grupo de jóvenes y personas mayores, siendo el grupo de personas mayores el que obtiene una mayor valoración $(M=16.28)$, frente al grupo de jóvenes $(M=10.80)$; y finalmente, en cuanto al estilo de compromiso, las diferencias se encuentran entre el grupo de adolescentes con adultos y personas mayores, de jóvenes con personas mayores, siendo el grupo de personas mayores la que obtiene una mayor puntuación $(M=16.57)$ frente a la adolescencia $(M=7.55)$.

En la tabla 4, se recogen los estilos de Gestión Conductual de los Conflictos en relación a la nacionalidad de las series de ficción. Como se puede observar, la distribución F de FisherSnedecor no muestra diferencias significativas en las diferentes escalas y la nacionalidad de las series.

Tabla 3

Prueba Sheffé para la variable Edad.

\begin{tabular}{|c|c|c|c|c|c|c|}
\hline & & Integración & Evitación & Dominación & Servilismo & Compromiso \\
\hline (I) EDAD & (J) EDAD & $p$ & $p$ & $p$ & $p$ & $p$ \\
\hline \multirow[t]{3}{*}{ Adolescencia } & Joven & .983 & .888 & .799 & .914 & .200 \\
\hline & Adultez & .073 & .000 & .000 & .140 & .002 \\
\hline & Vejez & .001 & .000 & .000 & .065 & .000 \\
\hline \multirow[t]{3}{*}{ Joven } & Adolescencia & .983 & .888 & .799 & .914 & .200 \\
\hline & Adultez & .326 & .011 & .000 & .075 & .574 \\
\hline & Vejez & .009 & .000 & .000 & .036 & .036 \\
\hline \multirow[t]{3}{*}{ Adultez } & Adolescencia & .073 & .000 & .000 & .140 & .002 \\
\hline & Joven & .326 & .011 & .000 & .075 & .574 \\
\hline & Vejez & .142 & .106 & .043 & .920 & .210 \\
\hline \multirow[t]{3}{*}{ Vejez } & Adolescencia & .001 & .000 & .000 & .065 & .000 \\
\hline & Joven & .009 & .000 & .000 & .036 & .036 \\
\hline & Adultez & .142 & .106 & .043 & .920 & .210 \\
\hline
\end{tabular}


Tabla 4

Estilos de Gestión Conductual del Conflicto en función de la nacionalidad de las películas.

\begin{tabular}{|c|c|c|c|c|c|c|c|c|}
\hline & & $n$ & $M$ & $D T$ & Mínimo & Máximo & $F$ & $p$ \\
\hline \multirow[t]{3}{*}{ Integración } & Española & 13 & 19.84 & 5.50 & 14.00 & 28.00 & .303 & .586 \\
\hline & No Española & 19 & 21.00 & 6.02 & 14.00 & 35.00 & & \\
\hline & Total & 32 & 20.53 & 5.75 & 14.00 & 35.00 & & \\
\hline \multirow[t]{3}{*}{ Evitación } & Española & 13 & 15.53 & 5.81 & 6.00 & 26.00 & .571 & .456 \\
\hline & No Española & 19 & 17.36 & 7.27 & 6.00 & 28.00 & & \\
\hline & Total & 32 & 16.62 & 6.68 & 6.00 & 28.00 & & \\
\hline \multirow[t]{3}{*}{ Dominación } & Española & 13 & 17.69 & 6.95 & 5.00 & 25.00 & 2.614 & .116 \\
\hline & No Española & 19 & 13.68 & 6.83 & 5.00 & 25.00 & & \\
\hline & Total & 32 & 15.31 & 7.06 & 5.00 & 25.00 & & \\
\hline \multirow[t]{3}{*}{ Servilismo } & Española & 13 & 12.92 & 3.32 & 6.00 & 18.00 & 1.601 & .215 \\
\hline & No Española & 19 & 14.52 & 3.64 & 6.00 & 18.00 & & \\
\hline & Total & 32 & 13.87 & 3.55 & 6.00 & 18.00 & & \\
\hline \multirow[t]{3}{*}{ Compromiso } & Española & 13 & 10.46 & 3.84 & 4.00 & 16.00 & 3.423 & .074 \\
\hline & No Española & 19 & 13.26 & 4.43 & 8.00 & 20.00 & & \\
\hline & Total & 32 & 12.12 & 4.36 & 4.00 & 20.00 & & \\
\hline
\end{tabular}

En la tabla 5 se detallan los resultados hallados en relación al género de las películas. Como se puede apreciar, existen diferencias estadísticamente significativas en las dimensiones de los diferentes estilos de gestión conductual del conflicto de evitación, dominación y compromiso.

Una vez aplicada la prueba Sheffé (tabla 6) se observan los siguientes resultados: en relación al estilo de gestión conductual de integración, aunque no se muestran diferencias estadísticamente significativas entre los grupos, la puntuación más alta aparece en el género del drama $(M=23.37)$, frente al valor más bajo que aparece en las series de ficción teen age $(M=16.37)$; en cuanto al estilo de gestión conductual de evitación, los resultados indican diferencias estadísticamente significativas entre las series de ficción teen age y las del género de la comedia y el drama, siendo el drama $(M=22.62)$ el que obtiene el mayor resultado en oposición a las series teen age $(M=10)$; en relación al estilo de gestión conductual de dominación, las diferencias halladas se encuentran entre los mismos grupos que el estilo anterior, siendo las series teen age $(M=22.50)$ las de mayor valor frente al género del drama $(M=10)$; en relación al estilo de gestión conductual de servilismo, no aparecen diferencias significativas entre ninguno de los grupos; y finalmente, en cuanto al estilo de compromiso, las diferencias se encuentran entre el grupo de las series de teen age y el género de la comedia y el drama, hallándose la puntuación más elevada en el género de la comedia $(M=14.66)$, y la menor en las series teen age $(M=8)$.

Tabla 5

Estilos de Gestión Conductual del Conflicto en función del género de las películas.

\begin{tabular}{|c|c|c|c|c|c|c|c|c|}
\hline & & $n$ & $M$ & $D T$ & Mínimo & Máximo & $F$ & $p$ \\
\hline \multirow[t]{5}{*}{ Integración } & Drama & 8 & 23.37 & 5.39 & 14.00 & 28.00 & 2.577 & .074 \\
\hline & Comedia & 6 & 22.33 & 9.13 & 14.00 & 35.00 & & \\
\hline & Teen age & 8 & 16.37 & 2.72 & 14.00 & 21.00 & & \\
\hline & Policiaca & 10 & 20.50 & 3.83 & 14.00 & 28.00 & & \\
\hline & Total & 32 & 20.53 & 5.75 & 14.00 & 35.00 & & \\
\hline \multirow[t]{5}{*}{ Evitación } & Drama & 8 & 22.62 & 5.26 & 13.00 & 28.00 & 8.499 & .000 \\
\hline & Comedia & 6 & 18.33 & 4.45 & 11.00 & 23.00 & & \\
\hline & Teen age & 8 & 10.00 & 4.69 & 6.00 & 17.00 & & \\
\hline & Policiaca & 10 & 16.10 & 5.54 & 7.00 & 23.00 & & \\
\hline & Total & 32 & 16.62 & 6.68 & 6.00 & 28.00 & & \\
\hline \multirow[t]{5}{*}{ Dominación } & Drama & 8 & 10.00 & 3.77 & 5.00 & 15.00 & 7.454 & .001 \\
\hline & Comedia & 6 & 12.50 & 8.21 & 5.00 & 25.00 & & \\
\hline & Teen age & 8 & 22.50 & 2.67 & 20.00 & 25.00 & & \\
\hline & Policiaca & 10 & 15.50 & 6.43 & 5.00 & 25.00 & & \\
\hline & Total & 32 & 15.31 & 7.06 & 5.00 & 25.00 & & \\
\hline \multirow{4}{*}{ Servilismo } & Comedia & 6 & 15.00 & 5.01 & 6.00 & 18.00 & & \\
\hline & Teen age & 8 & 12.00 & .00 & 12.00 & 12.00 & & \\
\hline & Policiaca & 10 & 13.80 & 2.89 & 12.00 & 18.00 & & \\
\hline & Total & 32 & 13.87 & 3.55 & 6.00 & 18.00 & & \\
\hline \multirow[t]{5}{*}{ Compromiso } & Drama & 8 & 14.00 & 3.70 & 8.00 & 20.00 & 4.905 & .007 \\
\hline & Comedia & 6 & 14.66 & 6.02 & 4.00 & 20.00 & & \\
\hline & Teen age & 8 & 8.00 & 2.13 & 4.00 & 12.00 & & \\
\hline & Policiaca & 10 & 12.40 & 2.95 & 8.00 & 16.00 & & \\
\hline & Total & 32 & 12.12 & 4.36 & 4.00 & 20.00 & & \\
\hline
\end{tabular}


Tabla 6

Prueba Sheffé de la variable género de ficción.

\begin{tabular}{|c|c|c|c|c|c|c|}
\hline & & Integración & Evitación & Dominación & Servilismo & Compromiso \\
\hline $\begin{array}{l}\text { (I) } \\
\text { Género }\end{array}$ & $\begin{array}{l}\text { (J) } \\
\text { Género }\end{array}$ & $p$ & $p$ & $p$ & $p$ & $p$ \\
\hline \multirow[t]{3}{*}{ Drama } & Comedia & .988 & .497 & .873 & 1.000 & .990 \\
\hline & Teen age & .102 & .000 & .001 & .420 & .029 \\
\hline & Policiaca & .736 & .085 & .247 & .914 & .844 \\
\hline \multirow[t]{3}{*}{ Comedia } & Drama & .988 & .497 & .873 & 1.000 & .990 \\
\hline & Teen age & .261 & .044 & .023 & .487 & .024 \\
\hline & Policiaca & .931 & .867 & .778 & .931 & .710 \\
\hline \multirow[t]{3}{*}{ Teen age } & Drama & .102 & .000 & .001 & .420 & .029 \\
\hline & Comedia & .261 & .044 & .023 & .487 & .024 \\
\hline & Policiaca & .465 & .119 & .092 & .762 & .127 \\
\hline \multirow[t]{3}{*}{ Policiaca } & Drama & .736 & .085 & .247 & .914 & .844 \\
\hline & Comedia & .931 & .867 & .778 & .931 & .710 \\
\hline & Teen age & .465 & .119 & .092 & .762 & .127 \\
\hline
\end{tabular}

\section{Discusión}

Del análisis de los datos obtenidos se observa una tendencia general por parte de los personajes principales de las series de ficción televisiva a la utilización de conductas de integración para la gestión de los conflictos. Por el contrario, el estilo menos frecuente es el compromiso, que según señalan Thomas (1992) y Van de Vliert (1997), se caracteriza porque las personas buscan el acuerdo a partir de concesiones mutuas pero sin preocuparse por maximizar los intereses de ambas partes. La integración, sin embargo, está determinada por la búsqueda de un acuerdo que satisfaga las aspiraciones de todos los implicados (De Dreu, Harinck y Van Vianen, 1999; Rahin y Magner, 1995), suponiendo conductas cooperativas con una actitud abierta al intercambio de información acerca de prioridades y preferencias, la puesta en común de ideas, la búsqueda de intereses compartidos y no divergentes. Este estilo integrativo es coincidente con el enfoque de la perspectiva única que propone la existencia de estilos más adecuados que otros independientemente de la situación, más específicamente, el estilo integrativo. De igual modo, y tal y como se proponía en la introducción teórica de este trabajo, el estilo integrativo, en el contexto social actual, es considerado expresamente adecuado desde un punto de vista moral, social y ético. Esto explicaría la utilización "ingenua" de este estilo, para significar el valor moral y acrecentar la deseabilidad social de los protagonistas y éticamente adecuada, generando características más atractivas, y por tanto, más persuasivas para el espectador.

También es posible hallar explicaciones a la utilización preferente del estilo integrativo apelando a la perspectiva contingente de gestión de conflictos, priorizando que cada uno de los estilos utilizados para gestionar el conflicto es apropiado en función de la situación. Desde este enfoque se plantea que utilizar la integración se considera adecuado cuando los conflictos tienen niveles elevados de intensidad o excesivo escalamiento, característica ésta muy frecuente en la construcción de los conflictos escenificados en las tramas de series de ficción cuyo desenlace suele "tener un final feliz", de resolución positiva del mismo.
En relación al sexo de los protagonistas, no se observan diferencias significativas en la forma en que los hombres y las mujeres tratan de resolver los conflictos en las series que protagonizan. Estos datos se corresponden con los estudios llevados a cabo sobre sujetos en situaciones reales de conflicto (Carnevale y Pruitt, 1992; Walter, Stulmacher y Meyer, 1998), en los que se evidencia que la concepción tradicional de atribuir más conductas cooperativas a mujeres y conductas competitivas a hombres no se corrobora con los resultados científicos, que más bien hablan de resultados inconsistentes en relación a esta variable.

En relación a la edad de los personajes analizados, se observa como las personas mayores presentan puntuaciones mayores en todos los estilos de gestión menos el de dominación en donde destacan los adolescentes. Como indica Medina (2006) el consumo de televisión exitoso conlleva la representación de temas universales como el conflicto y la comunicación entre padres e hijos, los modelos educativos de los hijos, etc., así como comportamientos estereotipados. De esta forma, el desenlace es previsible, cada personaje actúa y reacciona según el estereotipo que le caracteriza permitiendo que las personas anticipen el desenlace de la trama y generando una recompensa en el espectador. Así, el adolescente es representado con características más competitivas en donde el conflicto, tanto de lucha directa como indirecta, representa uno de los estereotipos más generalizados a nivel social.

En relación a la nacionalidad de las series de ficción, no se hallan diferencias entre las diferentes dimensiones del conflicto. Estos resultados encontrados, se explican a través de la teoría del cultivo que mantiene que la televisión propone mensajes que reflejan una realidad caracterizada por representaciones sociales universales (Lucas, García y Ruiz, 1999), que explicarían la uniformidad en los modos de pensar y actuar de los personajes de ficción televisiva, con independencia de la nacionalidad de la misma. Resulta evidente que gran parte de la producción de ficción televisiva presenta mensajes uniformes y una visión única del mundo, y muy habitualmente basadas en el modo de vida norteamericano. 
Finalmente, en relación a la gestión de conflictos presentados en diversos géneros de ficción analizados, se hallaron relaciones significativas cuando se trata de series dramáticas con el estilo evitativo. Por otro lado, también se halla una tendencia significativa del modo dominación cuando nos encontramos ante series dirigidas a público adolescente y juvenil. En el primer caso, es posible que la propia tensión dramática supedite a este tipo de estrategia que deja los conflictos deficientemente resueltos, y tiende a alargar y escalar el conflicto, concediendo más minutos e interés al discurso narrativo. En cambio, el estilo de dominación utilizado por el género de ficción juvenil, coincide con los códigos sociales y respuestas de este colectivo. Se trata de un estilo de gestión directo, explícito, poco elaborado y que permite visualizar al grupo de iguales quién tiene poder sobre quién.

Este trabajo ha pretendido analizar la importancia de los modelos de gestión de conflictos que se representan en las series de ficción, y que en gran medida, determinan muchas de las conductas imitadas por los jóvenes de nuestra sociedad. Sería necesario profundizar en esta línea para determinar de qué manera influyen y determinan los estilos de vida y la forma de afrontamiento de los conflictos en diferentes contextos.

\section{Referencias}

1. Ball-Rokeach, S. y DeFleur, M. (1976). A dependency model of mass media effects. Communication research, 3, 3-21. http://dx.doi.org/10.1177/009365027600300101

2. Bandura, A. (1977). Social Learning Theory. New York: General Learning Press.

3. Berkowitz, L. y Powers, P. C. (1979). Effects of timing and justification of witnessed aggression on the observers' punitiveness. Journal of Research in Personality, 13, 71-80. http://dx.doi.org/10.1016/0092-6566(79)90042-4

4. Bernárdez, A., García, I. y González, S. (2008). Violencia de género en el cine español. Análisis de los años 1998 a 2002 y guía didáctica. Madrid: Instituto de Investigaciones Feministas de la Universidad Complutense.

5. Bryant, J. y Zillmann, D. (2002). Media effects. Advance in Theory and Research. New Jersey: LEA.

6. Carrasco, A. (2010). Teleseries: géneros y formatos. Ensayo de definiciones, Miguel Hernández Communication Journal, 1, 174-200.

7. Carnevale, P.J. y Pruitt, D.G. (1992). Negotiation and Mediation, Annual Review of Psychology, 43, 531-582. http:// dx.doi.org/10.1146/annurev.ps.43.020192.002531

8. Cheung, C. y Chan, C. (1996). Televisión viewing and mean world value in Hong Kong's adolescents. Social Behavior and Personality, 24, 351-364. http://dx.doi.org/10.2224/ sbp.1996.24.4.351

9. De Dreu C.K.W., Harinck F. y Van Vianen A.E.M. (1999). Conflict and performance in groups and organizations. En C.L. Cooper y I.T. Robertson (Eds), Intenational Review of Industrial and Organizational Psychology (pp.376-405). Wiley: Chichester.

10. Del Río, P., Álvarez, A. y Del Río, M. (2004). Pigmalión. Informe sobre el impacto de la televisión en la infancia. Madrid: Fundación Infancia Aprendizaje.

11. Digón, P. (2006). El caduco mundo de Disney: propuesta de análisis crítico en la escuela. Comunicar. Revista científica de comunicación y educación, 26, 163-169.

12. Durkin, K. (1995). Developmental Social Psychology. Oxford: Blackwell.

13. Feilitzen, C. y Linne, O. (1975). Identifying with television characters. Journal of Communication, 25, 51-55. http:// dx.doi.org/10.1111/j.1460-2466.1975.tb00638.x

14. França, L. (2000). La contribución de las series juveniles de televisión a la formación de la identidad en la adolescencia. Análisis de contenido y de la recepción de la serie "Compañeros" (Antena 3). Tesis doctoral no publicada, Universidad Autónoma de Barcelona.

15. Gale, K. L. (2001). The sins and virtues of prime time television. Minnesota: University of Minnesota.

16. Galán, E. (2007). Construcción de género y ficción televisiva en España. Revista Comunicar, 28, 229-236.

17. García-Muñoz, N. y Fedele, M. (2011). Las series televisivas juveniles: tramas y conflictos en una «teen series». Revista Comunicar, 37, 133-140.

18. Guarinos, V. (2009). Fenómenos televisivos «teenagers»: prototipias adolescentes en series vistas en España. Coтunicar, 33, 203-211. http://dx.doi.org/10.3916/c33-2009-03$\underline{012}$

19. Gunter, B. (1986). Television and sex role stereotyping. London: Libbey.

20. Heintz-Knowles, K. (2000). Images of youth: A content analysis of adolescents in prime-time entertainment programming. Washington, DC: Frameworks Institute.

21. Hoyt, J. L. (1970). Effect of media violence "justification" on aggression. Journal of Broadcasting, 14, 455-464.

22. Huertas, A. (2002). La audiencia investigada. Barcelona: Gedisa.

23. Huston-Stein, A. y Frederick, L.K. (1975). Impact of television on children an youth. En E.M. Hetherington (Ed.), Review of child development research, 5 (pp. 183-256). Chicago: University of Chicago Press.

24. Jeffres, L. W. (1986). Mass Media process and effects. New York: Waveland Press.

25. Joy, L. A., Kimball, M. M. y Zabrack, M. L. (1986). Television and children's aggressive behavior. En T. M. Williams (Ed.), The impact of television: A natural experiment in three communities (pp. 303-360). New York: Academic Press.

26. Kimball, M.M. (1986). Television and sex-role attitudes. En T.M. Williams (Ed.), The impact of television: A natural experiment in three communities (pp.265-302). New York: Academic. 
27. Krcmar, M. y Cooke, M. C. (2001). Children's Moral Reasoning and Their Perceptions of Televisión Violence. Journal of Communication, 51, 300-316. http://dx.doi. org/10.1111/j.1460-2466.2001.tb02882.x

28. Lauzen, M. Dozier, D. y Horan, N. (2008). Constructing gender stereotypes through social roles in prime-time television". Journal of Broadcasting \& Electronic Media, 52, 200-214. http://dx.doi.org/10.1080/08838150801991971

29. López, J.A. y Cuenca, F.A. (2005). Ficción televisiva y representación generacional: modelos de tercera edad en las series nacionales. Comunicar, 25, 231-236.

30. Loscertales, F. y Nuñez, T. (2009). Familias y medios de comunicación. Sevilla: MAD.

31. Lucas, A., García, C. y Ruiz, J. A. (1999). Sociología de la Comunicación. Madrid: Trotta.

32. Martínez y Surinyac, G. (1998). El guión del guionista. Barcelona: CIMS.

33. Mastro, D.E. y Ortiz, M. (2008). A Content Analysis of Social Groups in Prime-Time. Spanish-Language Television. Journal of Broadcasting and Electronic Media, 52, 101118. http://dx.doi.org/10.1080/08838150701820916

34. Medina, M. (2006). Los mensajes de las series: eslóganes en imágenes. Comunicar, 26, 61-68.

35. Munduate, L., Ganaza, J. y Alcaide, M. (1993). Estilos de gestión del conflicto interpersonal en las organizaciones. Revista de Psicología Social, 8, 47-68.

36. Nimmo, D. (1978). Political Communication and Public Opinion in America, California: Goodyear Publishing Company.

37. Munduate, L., Ganaza, J. y Alcaide, M. (1993). Estilos de Gestión del Conflicto Interpersonal en las organizaciones. Revista de Psicología Social, 8, 47-68.

38. Palmer, E. L. y Dorr, A. (1980): Children and the Faces of Television. New York: Academic Press.

39. Pasquier, D. (1996). Teen Series'Reception: Televison, Adolescente and Culture of Feelings. Childhood: A Global Journal of Child Research, 3, 351-373.

40. Potter, W.J. (1990). Adolescents perceptions of the primary values of television Programming. Journalism Quarterly, 67, 843-853. http://dx.doi.org/10.1177/107769909006700439

41. Raffa, J. B. (1983). Television: The Newest Moral Educator. Phi Delta Kappan, 65, 214-215.

42. Rahim, M.A. (1983). Rahim Organizational Conflict Inventory-II: Forma $a$, $b$, and $c$. Palo Alto, CA: Consulting Psychologist Press.
43. Rahim, M.A. y Bonoma, T.V. (1979). Managing Organizational Conflict: A Model for Diagnosis and Intervention. Psychological Reports, 16, 143-155.

44. Rahim, M. A. y Magner, N. R. (1995). Confirmatory factor analysis of the styles of handling interpersonal conflict: First-order factor model and its invariance across groups. Journal of Applied Psychology, 80, 122-132. http://dx.doi. org/10.1037/0021-9010.80.1.122

45. Sahuquillo, P. (2007). Algunas aportaciones teóricas a la influencia de la televisión en el proceso de socialización de la infancia. Teoría de la educación, 19, 191-224.

46. Sánchez-Noriega, J.L. (2002). Historia del cine: teoría y géneros cinematográficos, fotografía y televisión. Madrid, Alianza.

47. Simelio, N. (2010). La representación de las relaciones sociales en las series de ficción digitales creadas especificamente para Internet. La televisión como contribución a la alfabetización digital. http://www.gabinetecomunicacionyeducacion.com.

48. Signorelli, N. y Bacue, A. (1999). Recognition and respect: A content analysis of primetime television characters across three decades. Sex Roles, 40, 527-544. http://dx.doi. org/10.1023/A:1018883912900

49. Thomas, K. W. (1992). Conflict and negotiation processes in organizations. In M.D. Dunnette y L.L. Hough (Eds.), Handbook of industrial and organizational psychology (pp. 651-717). Chicago: Rand McNally.

50. Thompson, J.B. (1998). Los media y la modernidad. Una teoría de los medios de comunicación. Barcelona: Paidós.

51. Toledano, G. y Verde, N. (2007). Cómo crear una serie de televisión. Madrid: T\&B Editores.

52. Van de Vliert, E. (1997). Complex Interpersonal Conflict Behavior. Psychology Press: London.

53. Walters, A. E., Stuhlmacher, A. F. y Meyer, L.L. (1998). Gender and negotiator competitiveness: A meta-analysis. Organizational Behavior and Human Decision Processes, 76, 1-29. http://dx.doi.org/10.1006/obhd.1998.2797

54. Ward, M. (2003). Understanding the role of entertainment media in the sexual socialization of American: A review of empirical research. Developmental Review, 23, 347-388. http://dx.doi.org/10.1016/S0273-2297(03)00013-3

Fecha de recepción: 11 de septiembre de 2012 Fecha de recepción de la versión modificada: 30 de octubre de 2012 Fecha de aceptación: 8 de noviembre de 2012 